no 63,063

\title{
THE PLASMAPAUSE AND ITS RELATION TO THE ION COMPOSITION IN THE TOPSIDE IONOSPHERE
}

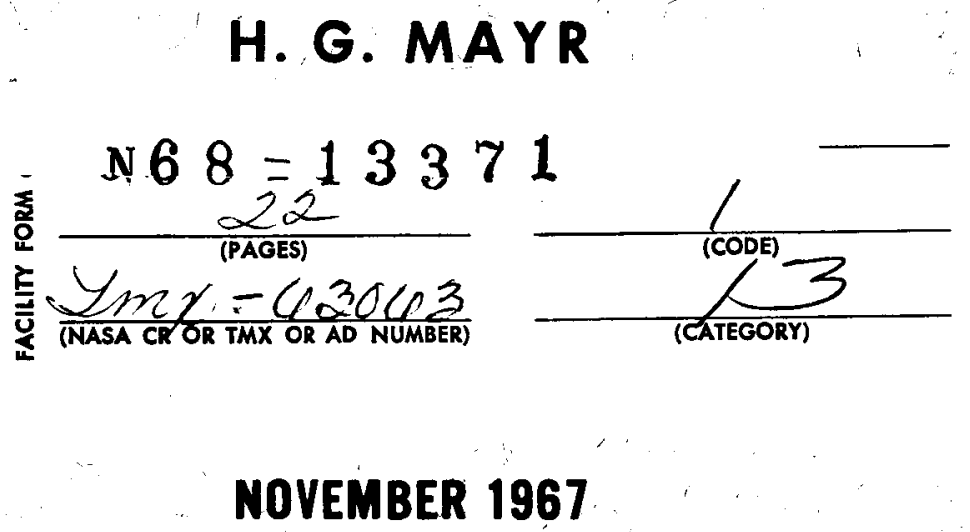

GODDARD SPACE FLIGHT CENTER GREENBELT, MARYLAND 
THE PLASMAPAUSE AND ITS RELATION TO THE ION COMPOSITION IN THE TOPSIDE IONOSPHERE

by

H. G. Mayr

Aeronomy Branch

GODDARD SPACE FLIGHT CENTER

Greenbelt, Maryland 


\begin{abstract}
The high $\mathrm{O}^{+}-\mathrm{H}^{+}$transition level at latitudes above 50 degrees and the plasmapause with its characteristic density plateau suggest that both features are strongly correlated; upward fluxes of protons can consistently account for both observations. It is shown that plasma escape can produce sufficiently large fluxes at latitudes where the field lines are open, thus significantly decreasing the hydrogen-ion density. As a result, plasma diffusion across field lines is induced-coupled with upward fluxes of ionization along field lines, which leads to the formation of a density plateau that characterizes the transition region from the open to the closed magnetosphere. Collision-type perpendicular diffusion would produce an enormously rapid transition over a distance of a few meters, which is not observed. This suggests that a turbulent state is built up in the transition region; thus, Bohm's coefficient for turbulent diffusion is employed, and this leads to a quantitatively satisfying description of the plasmapause.
\end{abstract}




\section{CONTENTS}

Abstract . . . . . . . . . . . . . . . . . . . . ii

INTRODUCTION .......................... . . . . 1

PHYSICAL CONCEPT . . . . . . . . . . . . . . . . . 2

General ....................... 2

Escape. . . . . . . . . . . . . . . . . 3

Diffusion Across Field Lines . . . . . . . . . . . . . . 3

THEORY . . . . . . . . . . . . . . . . . . . . 5

Continuity Equation . . . . . . . . . . . . . . . 5

Classical Diffusion . . . . . . . . . . . . . . . 11

Turbulent Diffusion . . . . . . . . . . . . . . 13

LIMITATIONS. . . . . . . . . . . . . . . . . . 15

References........................... 17 
The Plasmapause and its Relation to the Ion

Composition in the Topside Ionosphere

H. G. Mayr

\section{INTRODUCTION}

Whistler data [Carpenter, 1963, 1966] and ion-spectrometer measurements [Taylor et al., 1965] have shown that the electron concentration decreases abruptly and very rapidly above $L$ values of 3 to 5 . Recent observations from OGO satellite [Taylor, private communication] confirm this feature of the magnetosphere, which is referred to as the "plasmapause."

Mayr, Brace, and Dunham [1967] studied ion composition observed by Thomas et al. [1966] for the two major ion constituents $\mathrm{O}^{+}$and $\mathrm{H}^{+}$during minimum solar activity. They found that up to 50 degrees geomagnetic latitude the observed latitudinal temperature increase [Brace, Reddy, and Mayr, 1967] accounts for the increasing $\mathrm{O}^{+}-\mathrm{H}^{+}$transition level. At higher latitudes, however, the altitude of the observed transition level continues to increase; in contrast to theory, that predicts a slight decrease of this level. In order to explain this discrepancy, Mayr, Brace, and Dunham [1967] postulated that there are upward fluxes of ionization into the protonospheric field tubes that increase with latitude and thus significantly reduce proton concentration. In a static model such fluxes can arise only from ionization leakage out of the field tubes by diffusion across field lines. It was assumed that this was due to a density gradient across the magnetic field, the gradient being maintained through escape of protons at higher latitudes where the field line configuration permits it. This suggested that the density gradient across high-latitude field lines is related to the density plateau that constitutes the plasmapause. A consistent concept was thus proposed, combining the plasmapause at high altitudes with ion compositional features at high latitudes. 
This paper investigates this concept. Turbulent diffusion postulated by Bohm [1949], when combined with plasma escape, is shown to account quantitatively for the plasmapause and its relation to ion composition at high latitudes.

\section{PHYSICAL CONCEPT}

\section{General}

Describing the magnetosphere and considering the density structure of the dominant ion species, proton, we can distinguish three different regions in order of increasing altitude.

(1) The topside ionosphere. During minimum solar activity, the major ion constituents in this region (oxygen ions) are almost in diffusive equilibrium. The minor constituents (hydrogen ions) are in chemical equilibrium with $\mathrm{O}^{+}, \mathrm{O}$, and $\mathrm{H}$ through the resonance charge exchange reaction $\mathrm{H}+\mathrm{O}^{+} \rightleftarrows \mathrm{H}^{+}+\mathrm{O}$.

(2) The transition region between ionosphere and protonosphere. Here, diffusion processes become important for protons and comparable with chemical processes. In this most complex region, the $\mathrm{H}^{+}$concentration is very sensitive to the proton diffusion flux. As shown by Geisler and Bowhill [1965], upward fluxes of ionization, if in the order of the critical flux, can decrease the proton concentration by almost two orders of magnitude and correspondingly increase the transition level. Similarly, downward fluxes can significantly increase the $\mathrm{H}^{+}$concentration and lower the transition level.

(3) The protonosphere. Here, protons are the major constituents and can be considered in a state of fast diffusion along field lines, that is almost equivalent to diffusive equilibrium. The protonosphere has its roots in the previously described transition region. Any effect on the hydrogen-ion density there, due to diffusion fluxes, is shared by the entire density distribution along proton- 
ospheric field lines. The density in the protonosphere (although its variation along field lines corresponds almost to a diffusive equilibrium) is very sensitive to diffusion fluxes in the transition region.

With this understanding of the protonospheric density structure as it is coupled into the topside ionosphere, it is possible to outline a model of the plasmapause that is consistently correlated to observed ion composition in the topside ionosphere.

\section{Escape}

Figure 1 shows the field line configuration on the side of the earth [after Williams and Maed 1965] opposite to the sun. From this it is evident that the field lines become open at distances corresponding to approximately $L=6$, thus suggesting that beyond such distances plasma escape can be effective. An estimation based on a simple escape theory will show that escape fluxes must be on the order of the critical flux and can therefore significantly reduce the $\mathrm{H}^{+}$concentration. Thus one finds that the proton density tends to be much lower in the escape region than in the adjacent region where the field lines are closed and escape is impossible. As a result, a density gradient across field lines is built up, and diffusion across field lines forms a transition region between the "open" magnetosphere and the "closed" magnetosphere. We consider this transition region (shown in Figure 1 between the solid field lines) as the domain where the density plateau is formed that characterizes the plasmapause.

\section{Diffusion Across Field Lines}

From observations, in particular by Taylor et al. [1965], we can describe the density plateau as a rapid density decrease across field lines from $10^{3} \mathrm{~cm}^{-3}$ at $\mathrm{L}=4$, to $10 \mathrm{~cm}^{-3}$ at $\mathrm{L}=6$ in the equatorial plane, for instance). If as assumed, diffusion across field lines, induced by escape, is responsible for this feature, 


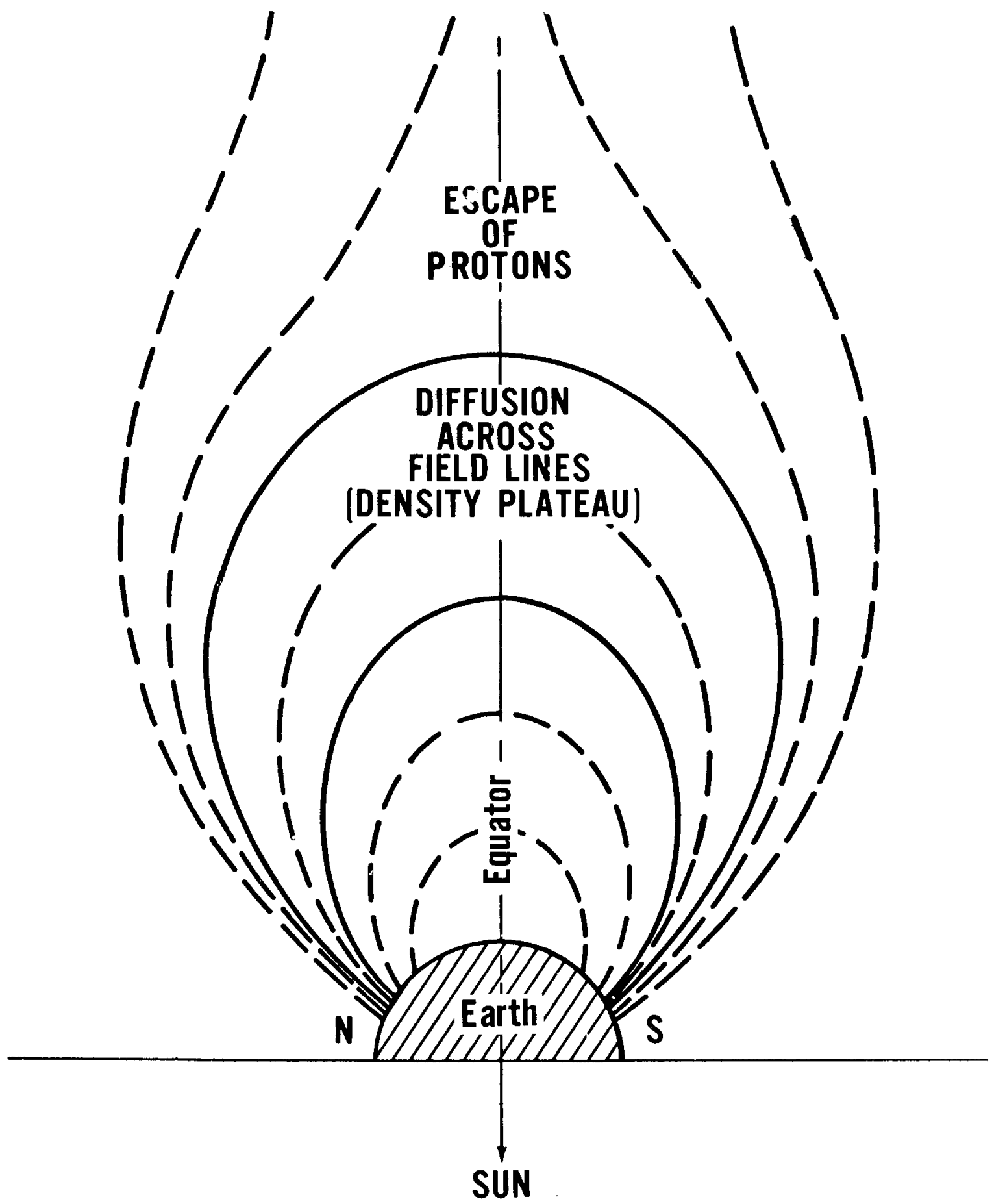

Figure 1. Field line configuration on the side of the earth opposite to the sun. 
then we must consider the following physical conception. The diffusion fluxes, and consequentily the density gradients across field lines, must be consistent with and coupled with the diffusion fluxes and density distributions along field lines.

Figure 2 shows the flux pattern of a field-line section near the equator, also an equatorial cross-section of the density plateau. The outward flow of ionization across the larger field line is compensated by an inward flow across the shorter field line and a diffusion flux inside the tube along the field lines. The convergence of the field tube enhances the flux density at lower altitudes, and this ionization flow in the transition region between ionosphere and protonosphere is responsible for the effect of the perpendicular diffusion. As pointed out previously, the parallel ionization flux in this region can significantly reduce the proton concentration in the field tube only if the flux is in the vicinity of the critical flux.

If escape and diffusion across field lines are the physical processes that form the plasmapause and its density plateau, then the resultant ionization fluxes parallel to the field lines must be on the order of the critical flux.

\section{THEORY}

\section{Continuity Equation}

This section presents an analytical treatment of the previously outlined concept. The density distribution in the protonosphere is described by the particle continuity equation which (under steady-state conditions) has the form:

$$
\operatorname{div} \vec{j}=\frac{1}{r^{2}} \frac{\partial}{\partial r}\left(r^{2} j_{r}\right)+\frac{1}{r \sin \theta} \frac{\partial}{\partial \theta}\left(\sin \theta j_{\theta}\right)+\frac{1}{r \sin \theta} \frac{\partial}{\partial \phi} j_{\phi}=0,
$$




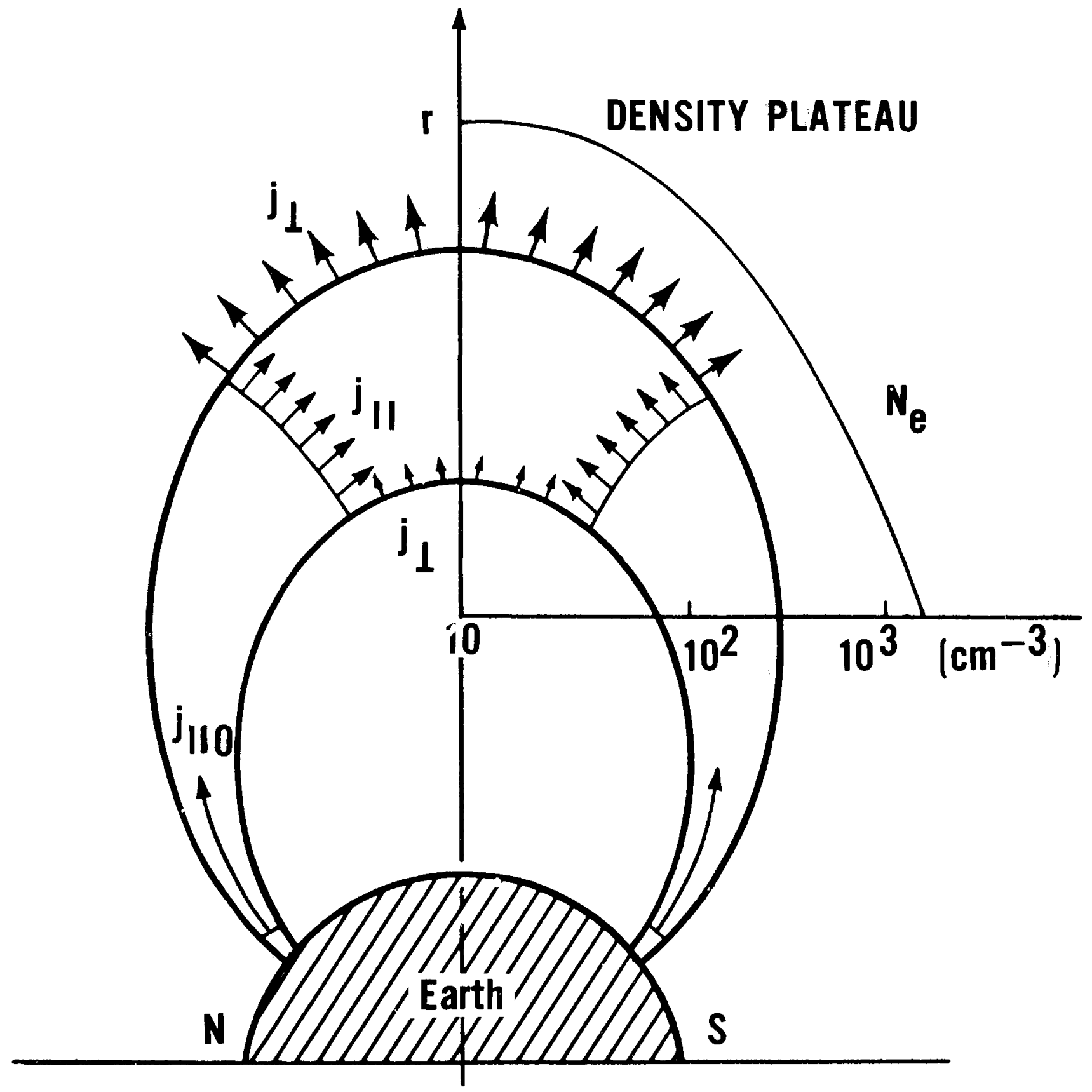

Figure 2. Flux pattern in a fieid tube. Superimposed is the shape of the density plateau. 
where $\vec{j}$ is the diffusion flux, and $r, \theta$, and $\phi$ are spherical polar coordinates. For the sake of simplicity, we confine our calculation to equatorial sections of field tubes (as shown in Figure 2). Neglecting the $\phi$-dependence in (1) leads then to

$$
\frac{1}{r^{2}} \frac{\partial}{\partial r}\left(r^{2} j_{\perp}\right)+\frac{1}{r} \frac{\partial}{\partial \theta} j_{11}=0
$$

where we introduced parallel and perpendicular diffusion fluxes

$$
\left(j_{\perp} \sim j_{r} ; j_{11} \sim j_{\theta}\right)
$$

as appropriate quantities in this near-equatorial region.

We assume that $j_{\perp}$ is almost independent of $\theta$ over the small region of concern and that $j_{11}=0$ at the equator, because of symmetry with respect to the equatorial plane. Then (2) leads to

$$
\frac{\partial}{\partial r}\left(r^{2} j_{\perp}\right)=\frac{r}{\theta} j_{1 \theta}
$$

where here $\theta$ is the latitude range over which we integrated (we assume $\theta$ to be 30 degrees), and $j_{{ }^{\prime} \theta}$ is the parallel flux at this latitude (shown in Figure 2).

As previously pointed out, the parallel diffusion flux at the bottom of the protonosphere can significantly affect the hydrogen-ion density. Therefore we transform the flux $j_{11 \theta}$ into the corresponding flux $j_{110}$ at the bottom of the tube by postulating that the total flux remains constant when going down along the field tube. This leads to the relation

$$
j_{1 \theta} \sim j_{10} \frac{r_{0}^{3}}{r^{3}} \frac{1}{\left(4-\frac{3 r_{0}}{r}\right)^{1 / 2}},
$$


where $r_{0}$ approximately equals the earth radius. Substituting (4) in (3) gives

$$
\frac{\partial}{\partial r}\left(r^{2} j_{\perp}\right)=j_{110} \frac{r_{0}^{3}}{\theta} \frac{1}{r^{2}\left(4-\frac{3 r_{0}}{r}\right)^{1 / 2}} .
$$

Equation 5 relates the diffusion flux across field lines to the parallel diffusion flux at the bottom of the protonosphere.

Geisler and Bowhill [1965] described in some detail the dependence of hydrogen-ion density on the diffusion flux $j_{110}$ in the transition region between ionosphere and protonosphere. At this level of our investigation we limit our argument by stating that the proton density in the protonosphere depends on the neutral-composition, oxygen-ion-density charge-exchange-rate coefficient, and, above all, on the transport flux $j_{10}$. By concentrating on the relation between $j_{110}$ and the proton density $N_{0}$, we can write $j_{110}=j_{110}\left(N_{0}\right)$, which, substituted in (5), yields

$$
\frac{\partial}{\partial r}\left(r^{2} j_{\perp}\right)=j_{110} N_{0} \frac{r_{0}^{3}}{\theta} \frac{1}{r^{2}\left(4-\frac{3 r_{0}}{r}\right)^{1 / 2}} .
$$

Equation 6 describes the coupling between the perpendicular diffusion flux $j_{\perp}$ (proportional to the density gradient across field lines) and the density at the bottom of the field tube; this density is coupled through diffusive equilibrium to the proton density in the equatorial region under discussion. We can give (6) a more physical meaning by saying that the net diffusion flux across a field tube is balanced by an upward flux of ions which, in its turn, affects the density in the tube itself.

Proton density is significantly affected only if the flux $j_{110}$ is near the critical flux. Then even a small variation in flux can change the proton density appreciably. Consequently, fluxes that can reduce the proton density so as to 
form the described density plateau must be on the order of the critical flux $j_{c}$, and we may therefore assume:

$$
\mathrm{j}_{110} \sim \mathrm{j}_{\mathrm{c}}=\text { constant } .
$$

With the assumption of (7), (6) can be integrated, which gives

$$
\left.\left.r^{2} j_{\perp}-r_{1}^{2} j_{\perp 1}=j_{c} \frac{4 r_{0}^{2}}{3 \theta}\right\}\left(1-\frac{3}{4} \frac{r_{0}}{r}\right)^{1 / 2}-\left(1-\frac{3}{4} \frac{r_{0}}{r_{1}}\right)^{1 / 2}\right\}
$$

At some inner field line at a distance $r_{1}$ we assume that the perpendicular diffusion process is attenuated, and therefore the density gradient corresponds to diffusive equilibrium.

Let us write for $j_{\perp}$ the expression

$$
j_{\perp}=-D(r \quad N) \frac{\partial N}{\partial r}
$$

where $\mathrm{D}(\mathrm{rN})$ is the general expression for the altitude and density-dependent diffusion coefficient. This leads to

$$
\left.D(r N) \frac{\partial N}{\partial r}=D\left(r_{1} N_{1}\right)\left(\frac{\partial N}{\partial r}\right)_{1}-j_{c} \frac{4 r_{0}^{2}}{3 \theta} \frac{1}{r^{2}} \mid\left(1-\frac{3}{4} \frac{r_{0}}{r}\right)^{1 / 2}-\left(1-\frac{3}{4} \frac{r_{0}}{r_{1}}\right)^{1 / 2}\right\}
$$

which describes the density variation across field lines in a near-equatorial region.

In order to solve (10) we must know the density $\mathrm{N}_{2}$ at some distance $\mathrm{r}_{2}$. We assume that plasma escape is what induces diffusion across field lines and thus forms the density plateau. The boundary value $\mathrm{N}_{2}$ for solving (10) must 
therefore be chosen as the density in the escape region which begins at a distance of $r_{2} \sim 6 r_{0}$.

A very simple estimation of the escape effect leads to an approximate evaluation of $\mathrm{N}_{2}$. Following Jastrow and Rasool [1965], we find that the density at the escape level is less than $10^{5} \mathrm{~cm}^{-3}$. Thus $\mathrm{H}^{+}$must escape immediately above the charge exchange region. (somewhere between 500 and $1000 \mathrm{~km}$ during minimum solar activity). With temperatures on the order of $3000^{\circ} \mathrm{K}$ [Brace, Ready, and Mayr, 1967] the escape flux $\mathrm{j}_{\mathrm{e}}$ is then related to the proton density in the ion composition transition region through

$$
\mathrm{j}_{\mathrm{e}} \sim 10^{6} \times \mathrm{N}_{20}
$$

where the subscript 2 again refers to the escape region. Obviously the upper limit of $\mathrm{N}_{20}$ is determined by the highest possible flux, the critical flux $\mathrm{j}_{\mathrm{C}}$. Assuming this flux to be on the order of the critical flux (assumed to be $\mathrm{j}_{\mathrm{e}} \sim 10^{7} \mathrm{~cm}^{-2} \mathrm{sec}^{-1}$ ), we find a value $\mathrm{N}_{c 0} \sim 10^{2} \mathrm{~cm}^{-3}$, that is much lower than the diffusive equilibrium value. Conversely, if flux $j_{e}$ is appreciably smaller than the critical flux, it will not very much affect the $\mathrm{H}^{+}$density; thus $\mathrm{N}_{20}$ will approach its diffusive equilibrium value, which cannot satisfy (11). Therefore, $\mathrm{j}_{\mathrm{e}}$ must be in the vicinity of the critical flux, and $\mathrm{N}_{20}$ is on the order of $10^{2} \mathrm{~cm}^{-3}$ because of the escape of ionization.

Assuming that the protons in the escape region are still in a state of fast diffusion and therefore almost in diffusive equiribrium, we find then that the hydrogen-ion density $\mathrm{N}_{2}$ is on the order of $\mathrm{N}_{2} \sim 10 \mathrm{~cm}^{-3}$ at $\mathrm{r}_{2}=6 \mathrm{r}_{0}$. After thus having determined the boundary conditions, (10) can be solved. In later portions of this paper we derive the density distributions for two kinds of diffusion mechanisms and discuss the results in the light of plasmapause manifestations. 
Classical Diffusion

The term "classical diffusion" refers to plasma drift across field lines, caused by collisions between electrons and ions. Spitzer [1956] shows that the perpendicular diffusion coefficient of the plasma is

$$
D_{c}=7.2 \times 10^{-2} \times \frac{N}{T^{1 / 2} B^{2}}
$$

where $D_{c}$ is the classical coefficient; $N$ is the density; $B$ is the magnetic field; and $\mathrm{T}$ is the plasma temperature.

Substituting for the magnetic field in (12), the equatorial dependence (corresponding to the region we are concerned with)

$$
B=\frac{0.3 r_{0}^{3}}{r^{3}}
$$

and adopting from Serbu and Maier [1965] the observed temperature dependence as

$$
\mathrm{T} \sim 5 \times 10^{2} \frac{\mathrm{r}^{2}}{\mathrm{r}_{0}^{2}}
$$

leads to

$$
D_{c}=3.5 \times 10^{-2} \frac{\mathrm{r}^{5}}{\mathrm{r}_{0}^{5}} \mathrm{~N}
$$

Substituting this diffusion coefficient in (10) gives

$$
\frac{\partial N^{2}}{\partial r}=\left(\frac{\partial N^{2}}{\partial r}\right)_{1} r_{1}^{5}-j_{c} \frac{80}{6}\left(\frac{r_{0}}{r}\right)^{7}\left\{\left(1-\frac{3}{4} \frac{r_{0}}{r}\right)^{1 / 2}-\left(1-\frac{3}{4} \frac{r_{0}}{r_{1}}\right)^{1 / 2}\right\}
$$


which can be integrated, thus leading to

$$
\begin{aligned}
N^{2}=N_{0}^{2}-\left(\frac{\partial N^{2}}{\partial r}\right)_{1} & \frac{r_{1}}{4}\left[\left(\frac{r_{1}}{r}\right)^{4}-\left(\frac{r_{1}}{r_{2}}\right)^{4}\right] \\
& -j_{c} \frac{80}{\theta} r_{0}\left\{\left(\frac{r_{0}}{r}\right)^{6}\left[\frac{1}{6}\left(1-\frac{3}{4} \frac{r_{0}}{r_{1}}\right)^{1 / 2}-\frac{2}{13}\left(1-\frac{3}{4} \frac{r_{0}}{r}\right)^{1 / 2}-\frac{1}{78}\right]\right\}
\end{aligned}
$$

The boundary conditions can be summarized as follows:

For

$$
r_{2}=5.5 \times r_{0}, N_{2}=10 / \mathrm{cm}^{-3}
$$

and at

$$
r_{1},\left(\frac{\partial N}{\partial r}\right)=\left(\frac{\partial N}{\partial r}\right)_{1}=\frac{N_{1}}{H}
$$

with $\mathrm{H}$ the density scale height of the closed, undisturbed magnetosphere. Applying these conditions to (14) leads to a density distribution shown in Figure 3 (dotted line). A closer examination of (14) reveals that the transition from the escape region (with a density of $10 \mathrm{~cm}^{-3}$ ) to the almost unperturbed "closed" magnetosphere (with its observed concentration in the order of $10^{3} \mathrm{~cm}^{-3}$ ) is extremely sharp, occurring over a distance of only a few meters. The diffusion coefficient used is so small that a huge density gradient is needed to consistently affect the proton distributions along field lines. A comparison of the computed density gradient with the observed density distribution by Taylor et al. [1966] for the equatorial region indicates a striking disagreement especially when we consider that the observed transition region occurs over a distance of almost two earth radii. 
Turbulent Diffusion

Failure to describe the plasmapause with the collision-type diffusion raises the question whether another diffusion mechanism could possibly account for the observed density plateau. Bohm [1949] found experimentally that the perpendicular diffusion became enormously enhanced for a plasma in turbulent state, and he verified this effect theoretically. Several authors [Braginskii, 1965, Kautman, 1966, and Spitzer, 1966] have since confirmed this plasma property.

It is plausible that the sharp transition from the closed to the open magnetosphere that is to be expected for collision-type diffusion should cause turbulence in the magnetosphere. We thus used Bohm's diffusion coefficient

$$
D_{B} \sim 10^{3} \frac{T}{B}[\text { Spitzer, } 1956]
$$

in our attempt to describe the plasmapause.

If we introduce into (17) the already quoted temperature and magneticfield variations, (10) leads then to the solution

$$
\begin{aligned}
& \mathrm{N}=\mathrm{N}_{2}-\left(\frac{\partial \mathrm{N}}{\partial \mathrm{r}}\right)_{1} \frac{\mathrm{r}_{1}}{4}\left[\left(\frac{\mathrm{r}_{1}}{\mathrm{r}}\right)^{4}-\left(\frac{\mathrm{r}_{1}}{\mathrm{r}_{2}}\right)^{4}\right] \\
&-\mathrm{j}_{\mathrm{c}} \frac{4 \times 10^{-6}}{5 \theta} \mathrm{r}_{0}\left(\left(\frac{\mathrm{r}_{0}}{\mathrm{r}}\right)^{-6}\left[\frac{1}{6}\left(1-\frac{3}{4} \frac{\mathrm{r}_{0}}{\mathrm{r}_{1}}\right)^{1 / 2}-\frac{2}{13}\left(1-\frac{3}{4} \frac{\mathrm{r}_{0}}{\mathrm{r}}\right)^{1 / 2}-\frac{1}{78}\right]\right. \\
&\left.-\left(\frac{\mathrm{r}_{0}}{\mathrm{r}}\right)^{6}\left[\frac{1}{6}\left(1-\frac{3}{4} \frac{\mathrm{r}_{0}}{\mathrm{r}_{1}}\right)^{1 / 2}-\frac{2}{13}\left(1-\frac{3}{4} \frac{\mathrm{r}_{0}}{\mathrm{r}_{2}}\right)^{1 / 2}-\frac{1}{78}\right]\right)
\end{aligned}
$$

to which we apply the boundary conditions in (15) and (16). The result is shown in Figure 3 (solid, thick line) and reveals an acceptable agreement with the observed density distribution (solid, thin line). 


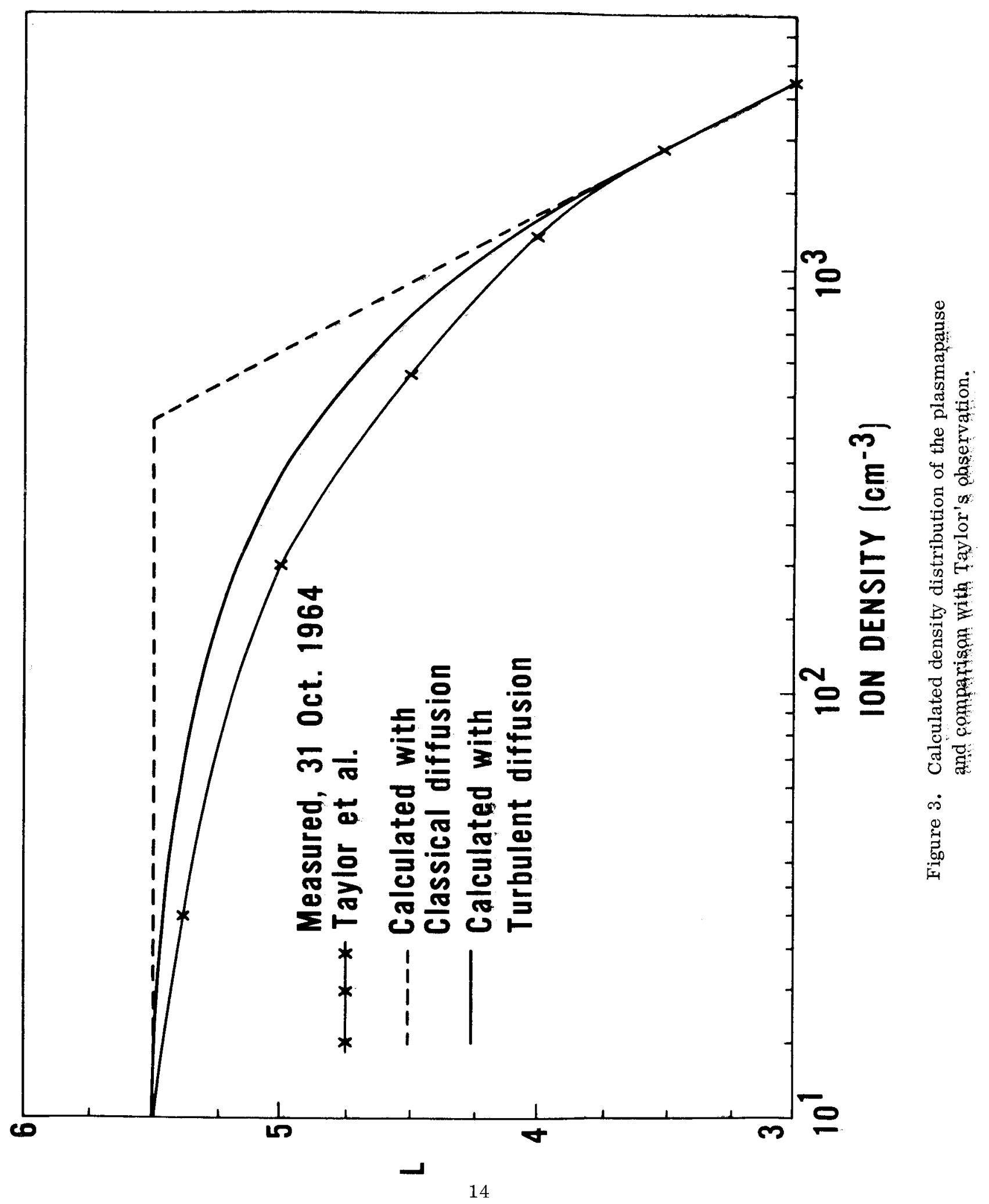


It should be noted that this conformity was achieved by choosing the boundary level $r_{1}$ properly at $L=5.5$; thus, we assumed that the field lines become open at this distance. As shown by Taylor et al. [1966], the location of the plasmapause varies strongly with changing solar activity. We assume that this is due to variations in the field-line structure, such that there are variations in the location where the field lines become open. The distribution shown in Figure 3 should therefore be characterized by its shape and the approximate range of the density plateau in the transition region from the open to the closed magnetosphere, and not by its absolute location.

We conclude that plasma escape in combination with turbulent diffusion across field lines is a valid concept for explaining the plasmapause with its characteristic density plateau. The resulting upward fluxes of ionization can evidently also account for the unexpectedly high ion-composition transition level at geomagnetic latitudes above 50 degrees.

\section{LIMITATIONS}

A rigorous treatment of the described concept would involve the simultaneous solution of the nonlinear, (at least) two-dimensional and time dependent continuity equations for the two major ion constituents of protonosphere and topside ionosphere. In order to avoid this difficult task séveral simplifying assumptions and approximations were made which have to be discussed.

The basic assumption was that the plasmapause is produced by the escape of ionization into the magnetic tail on tise night side of the earth. Taylor's measurements indicate that the plasmapause similarly exists also on the day side where the field lines are closed; so escape cannot be directly responsible. Evidently, the magnetosphere is in a highly dynamic state. With proton fluxes that cannot exceed the critical flux $\mathrm{j}_{\mathrm{c}} \sim 10^{7} / \mathrm{cm}^{-2} \mathrm{sec}^{-1}$, a protonospheric field 
tube with $1 \mathrm{~cm}$ square basis area can only be replenished by a particle population on the order of $10^{11}$ during half a day. This amount is smaller by almost two orders of magnitude than the proton content to be expected on the basis of diffusive equilibrium. Thus it cannot significantly distort the distribution induced by escape; therefore, even on the day side, the typical picture of the plasmapause prevails.

Furthermore, our analysis considered only the equatorial region. This was in order to make certain simplifying assumptions concerning the geometry of the field tube and the magnetic field itself, leading to an analytical solution. As a consequence, the ionization flux at the bottom of the protonosphere resulted only from a part of the field tube arid is therefore somewhat low. This is so far not a serious limitation as other uncertainties imply deviations that could easily compensate for this approximation. These uncertainties concern the critical flux, which is not well determined because of our ignorance of the value of the charge-exchange coefficient; they concern Bohm's diffusion coefficient, which is considered as known within one order of magnitude.

The various experimental evidences of the plasmapause are far from being consistent; thus the plasmapause and its form are not at all well established. The same is true of our concept. It is justified chiefly by the agreement between our theoretical result and Taylor's ion spectrometer measurements on OGO. We, in turn, can offer an explanation only for this type of observation. We cannot explain Serbu and Maier's measurements on IMP [1966], which do not suggest a density plateau, but rather a recovery of the plasma density in a region where we regard plasma escape as significant.

\section{ACKNOWLEDGEMENTS}

I gratefully acknowledge the encouraging discussions with $\mathrm{H}$. A. Taylor and the helpful comments by L. H. Brace, S. Chandra, and J. G. C. Walker. 


\section{REFERENCES}

Bohm, D., The characteristics of electrical discharges in magnetic fields, Ed. A. Guthrie and R. K. Wakering, 62-65, ivícGraw-Hill Book Co., New York, 1949.

Braginskii, S. I., Transport processes in a plasma, Ed. M. A. Leontovich, 235-236, Consultants Bureau, New York, 1965.

Brace, L. H., B. M. Reddy, and H. G. Mayr, Global behaviour of the ionosphere at $1000 \mathrm{~km}$ altitude, J. Geophys. Res., 72, 1967.

Carpenter, D. L., Whistler evidence of a "knee" in the magnetospheric ionization density profile, J. Geophys. Res., 68, 1675-1682, 1963.

Carpenter, D. L., Whistler studies of the plasmapause in the magnetosphere, 1, Temporal variation in the position of the knee and some evidence on plasma motions over the knee, J. Geophys. Res., 71, 693-710, 1966.

Geisler, J. E. and S. A. Bowhill, An investigation of ionosphere-protonosphere coupling, Aeronomy Report No. 5, University of Illinois, Urbana, 1965.

Jastrow R., and S. I. Rasool, Introduction to Space Science, Ed. W. N. Hess, Gordon and Breach Science Publishers, 1965.

Kaufman, A. N., Dissipative effects in plasma physics in theory and application, W. B. Kinke1, Ed. McGraw-Hill Book Co., New York, 1966, 92.

Mayr, H. G., L. H. Brace, and G. S. Dunham, On the ion composition and temperature in the topside ionosphere, J. Geophys. Res. 72, 1340-1352, 1967. 
Serbu, C. P., and E. J. R. Maier, Low-energy electrons measured on IMP 2, J. Geophys. Res. 71, 3755-3766, 1966.

Spitzer, L., Jr., Physics of fully ionized gases, Interscience Publishers, Inc., New York, 1956.

Taylor, H. A., Jr., H. C. Brinton, and C. R. Smith, Positive ion composition in the magnetosphere obtained from OGOA satellite, J. Geophys. Res., 70, 5769-5791, 1965.

Thomas, J. O., M. J. Rycroft, L. Colin, and K. L. Chan, Electron density profiles in ionosphere and exosphere, proceedings of the Nato Advanced Study Institute held at Finse, Norway, Edit. Jon Frihagen, North-Holland Publishing Company, Amsterdam, 1966.

Williams, D. J., and G. D. Mead, Nightside magnetosphere configuration as obtained from trapped electrons at 1100 kilometers, J. Geophys. Res., 70, 3017-3029, 1965. 